\title{
Video Watermarking for Persistent and Robust Tracking of Entertainment Content (PARTEC)
}

\author{
Deepayan Bhowmik ${ }^{1}$, Charith Abhayaratne ${ }^{2}$ and Stuart Green ${ }^{3}$ \\ 1 Division of Computing Science and Mathematics, University of Stirling, Stirling, UK \\ 2 Dept of Electronic and Electrical Engineering, University of Sheffield, Sheffield, UK \\ 3 ZOO Digital Group plc, Sheffield, UK \\ deepayan.bhowmik@stir.ac.uk, c.abhayaratne@sheffield.ac.uk, \\ stuart.greenezoodigital.com
}

\begin{abstract}
The exploitation of film and video content on physical media, broadcast and Internet involves working with many large media files. The move to filebased workflows necessitates the copying and transfer of digital assets amongst many parties, but the detachment of assets and their metadata leads to issues of reliability, quality and security. This paper proposes a novel watermarking based approach to deliver a unique solution to enable digital media assets to be maintained with their metadata persistently and robustly. Watermarking based solution for entertainment content manifests new challenges, including maintaining high quality of the media content, robustness to compression and file format changes and synchronisation against scene editing. The proposed work addresses these challenges and demonstrates interoperability with existing industrial software framework for media asset management (MAM) systems.
\end{abstract}

\section{Introduction}

Digital watermarking received significant attention in recent past for various multimedia related applications such as copyright protection protection, image quality monitoring or media integrity verification. For example, Cox et al. [7] identified broadcast monitoring, owner identification, proof of ownership, authentication, transactional watermarking, copy control and covert communication as potential watermarking applications of which some are adopted in the industry ${ }^{4}$ with additional applications such as audience measurement or improved auditing. Among other applications (1) image quality measurement was proposed in [10] by measuring the degradation of the extracted watermarking; (2) [12] proposed a watermarking method for improved management of medical records through embedding patient data such as identity, serial number or region of interest to ensure the image association with the correct patient; or (3) a real-time system for video-on-demand services where frame images are watermarked, unique to the user and aims to deter piracy [15]. On contrary this paper considers a new application of watermarking in tracking entertainment content from production to post processing to distribution. In designing our new algorithm it is important to revisit the literature that looked at various characteristics of watermarking algorithms including algorithms that are either imperceptible [1], robust to intentional [8] and unintentional (e.g., compression [11], filtering or geometric [16]) attacks or fragile [6] or secure [4].

\footnotetext{
${ }^{4}$ www.digitalwatermarkingalliance.org
} 
This work proposes a new video watermarking application for Persistent and Robust Tracking of Entertainment Content (PARTEC). PARTEC is concerned with improving the assets and their metadata management in file based workflows required for reliably copying and transferring digital assets amongst many parties. The exploitation of film and video content on physical media, broadcast and Internet involves working with many large media files. Because of the size of media files, they are inevitably copied multiple times during post production so that operators and different physical locations can work efficiently by having a local copy. This means that it is not practical to record the assets in one central location from which multiple operators have common access. A consequence of this is that multiple copies exist and therefore there is difficulty in maintaining integrity of those copies. For example, if a change is made to the master copy, then there is currently no simple way for that change to be propagated to all copies, i.e., version control.

Detachment of assets and their metadata leads to issues of reliability, quality and security in media post processing industry. PARTEC delivers a new, unique watermarking based solution to enable digital media assets to be maintained and tracked with their metadata persistently and robustly by embedding unique identifiers as watermark. Additionally the proposed solution also enables personalisation of each copy of an asset file and so affords a level of security to prevent unauthorised access to protected data. However, watermarking entertainment content manifests new challenges, such as, $a$ ) limited / no distortion (due to embedding) is permissible for high resolution and high quality studio contents; $b$ ) the solution must be compatible with existing industrial file formats; $c$ ) robust to reasonable compression and format changes; and $d$ ) robust to scene editing, i.e., inclusion or exclusions of frames within existing content, joining multiple clips to create a new edit or producing multiple clips from a single source. Our work proposes a solution that addresses the above mentioned challenges and demonstrates interoperability with existing industrial software framework for media asset management (MAM) systems. Main contributions of our work are:

- A unique watermarking-based system for persistent and robust tracking of entertainment content,

- New watermarking algorithms suitable for frame domain, MPEG-2 and H.264 compression domain embedding with high imperceptibility and

- Techniques for watermark identifier extraction robust to scene editing, media file format changes and compression.

\section{Motivation and Requirements Analysis}

PARTEC considers wider industry requirements from the users including $a$ ) media companies producing film and video content; $b$ ) content post-processing companies providing professional services to entertainment markets; and $c$ ) developers of MAM systems for the entertainment industry. Application scenarios include a media company wishes to offer paid-for video-based educational resources which are available to consumers across the world through its video streaming website. Updatable metadata is a critical component of these materials, is time sensitive and easily detached from the media 


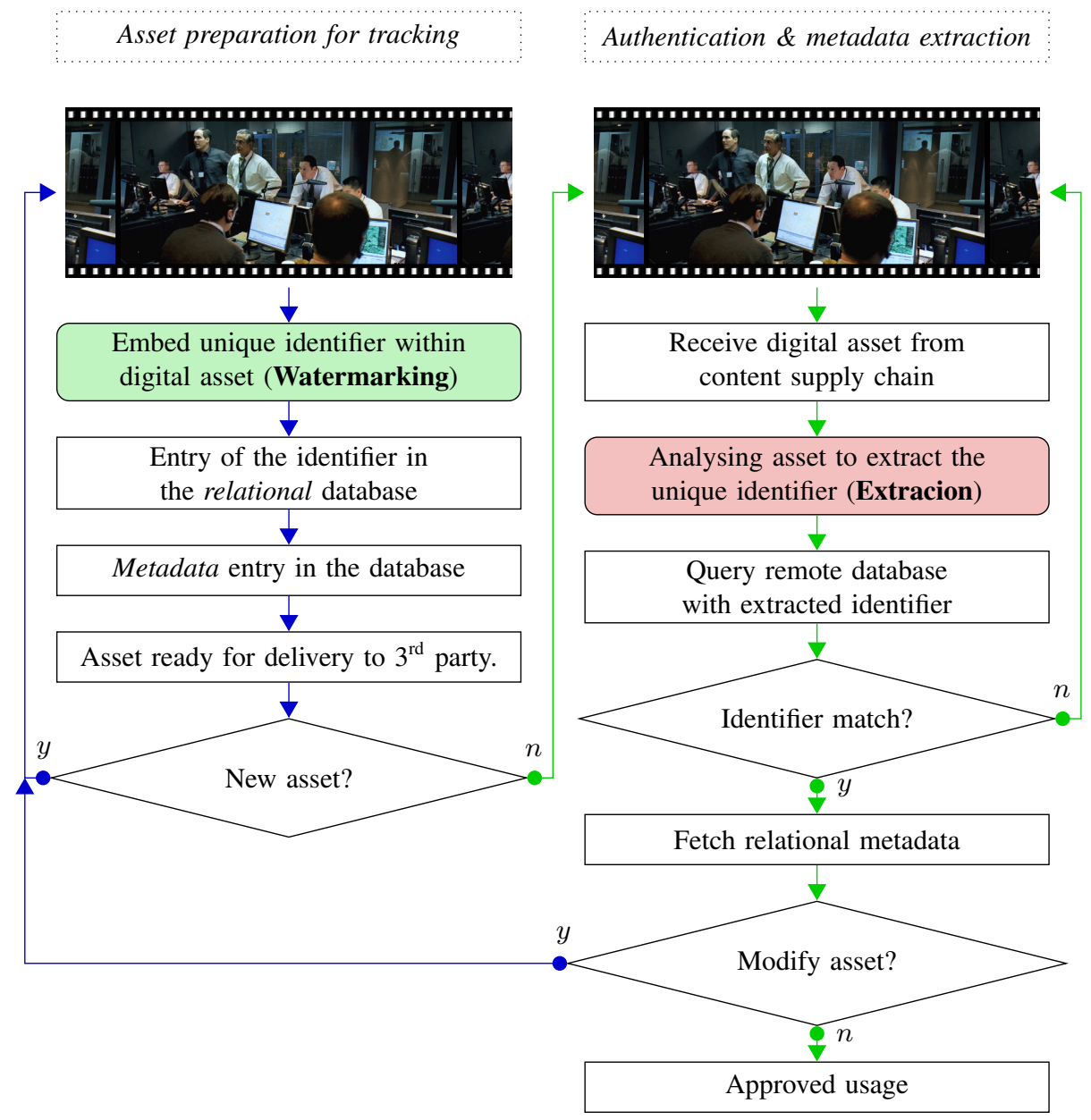

Fig. 1. PARTEC overall system diagram.

when teachers download and create copies, thereby diminishing value. Our solution ensures the persistent association of media with the associated metadata throughout commercialisation of the media content. The approach delivers the very latest metadata on each occasion when a media asset is accessed and enables authentication of file based assets by validating their contents, version, the associated access permissions, rights clearances and other metadata including any revisions to an asset's metadata.

PARTEC represents a new application of watermarking techniques for the purpose of asset tracking, authentication and security. To the best knowledge of the authors there exists no solution that addresses this scope or that applies imperceptible watermarking techniques for the purposes envisaged, having consulted with significant content producers and assessing the product specifications of MAM systems. As a significant shift towards file-based workflows in the entertainment industry mandates for greater lev- 


\begin{tabular}{l|l}
\hline Requirements & \multicolumn{1}{c}{ Description } \\
\hline Media format & $\begin{array}{l}\text { Statistical analysis of the available contents in terms of file type / wrapper } \\
\text { format and video stream formats, i.e., compression / encoding types. }\end{array}$ \\
\hline Imperceptibility & $\begin{array}{l}\text { Requirements for allowable quality degradation after watermark embed- } \\
\text { ding. }\end{array}$ \\
\hline Robustness & $\begin{array}{l}\text { Robustness of the algorithm that successfully preserve the watermark } \\
\text { identifier under various compression and format changes. }\end{array}$ \\
\hline Synchronisation & $\begin{array}{l}\text { Ability to retrieve and identify the content which were modified such as } \\
\text { scene editing, frame dropping, joining multiple clips etc. }\end{array}$ \\
\hline
\end{tabular}

Table 1. Types of industrial requirements.

els of tracking and security, our approach is timely and solves an existing industrial problem. An overall system diagram is depicted in Fig. 1 and this paper describes the watermarking based solution (coloured boxes) which is the central theme of PARTEC.

\subsection{Requirements analysis}

As the part of the system design we analyse the requirements with greater understanding of the approaches currently taken in the industry for media asset management. We also gave better emphasis on the interoperability of the proposed system with existing framework as this is necessary for industrial adaptation. The requirements analysis is dissected in four different categories as discussed below and shown in Table 1.

Media format A solution capable of handling existing file types is important to the media industry. In PARTEC we deal with two different categories of file types:

- Audio-video wrapper formats that multiplex video streams with optional audio streams and sub-title text and to provide one single file type (e.g., DAT, VOB, MOV, AVI, MP4, MPG etc.).

- Video stream formats, available within the wrapped files mentioned above. These file types are encoded video bit-streams complying either proprietary (e.g., ProRes by Apple) or international standards, e.g., MPEG-2, H.264 etc.

In order to achieve maximum coverage, we have analysed a statistically representative sample consisting of 168, 389 media files available from industrial partners' asset repositories, of which major wrapper types account for DAT: $43 \%$, VOB: $21 \%$, MOV: $15 \%$ and AVI: $13 \%$. With respect to video stream formats, MPEG- 2 occupies $47 \%$ of the total repository, followed by RAW (17\%), H.264 (9\%), ProRes (6\%) and the remainder is made up of other formats. We have considered these statistics in designing PARTEC.

Imperceptibility Imperceptibility (visual quality) and robustness are widely considered as the two main properties vital for a good digital watermarking system. They are complimentary to each other and hence challenging to attain the right balance between them. However, in processing entertainment media, the visual quality carries significant weight to provide highest Quality-of-Experience (QoE). Our design is heavily influenced by this requirement and ensures imperceptibility after watermark embedding. 


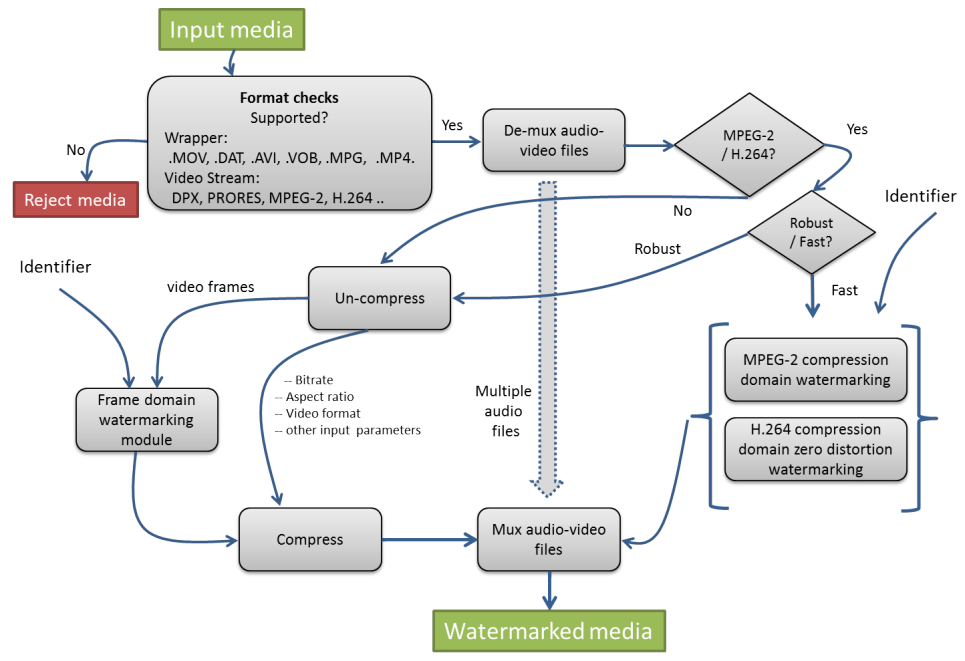

Fig. 2. Overall embedding work-flow including media format handling watermark embedding.

Robustness The requirement demands two different types of robustness of the extracted watermark, $a$ ) robustness against video format change and $b$ ) robustness to rational compression using popular standards, i.e., MPEG-2 and H.264 here. These are needed to support a change in format or compression ratio during post production, i.e., preparing screening quality, DVD quality or other types of content.

Synchonisation Synchronisation is one of the major issues within video watermarking domain that is rarely addressed. In PARTEC, the synchronisation problem emerges due to media post production including scene editing, frame dropping, combining multiple clips or inserting frames within a clip. A solution should be capable of identifying clips from multiple sources or multiple segments within a single clip. Watermark synchronisation (at least) at frame level is an essential component of our proposed solution.

\section{The System Architecture}

PARTEC system architecture fulfils the industrial requirements with three major functional modules: $a$ ) file format handling, $b$ ) watermark embedding and extraction and $c$ ) synchronisation. The overall flow diagram for embedding is shown in Fig. 2.

\subsection{File format handling}

Compatibility of the solution with existing media file types is important in industry. As discussed earlier, two types of media files need to be handled in PARTEC: $a$ ) audiovideo (AV) wrappers and $b$ ) video stream formats. Our strategy is to demultiplex the AV wrapper to separate the video and audio stream. While we process the video stream for watermarking, we keep the audio stream in a temporary file. After embedding, we remultiplex the watermarked video stream with the temporarily stored audio to produce a watermarked file in the same AV format as originally received. The file format handling module also checks for supported formats, i.e., MOV, VOB, MPG, MP4 etc. 


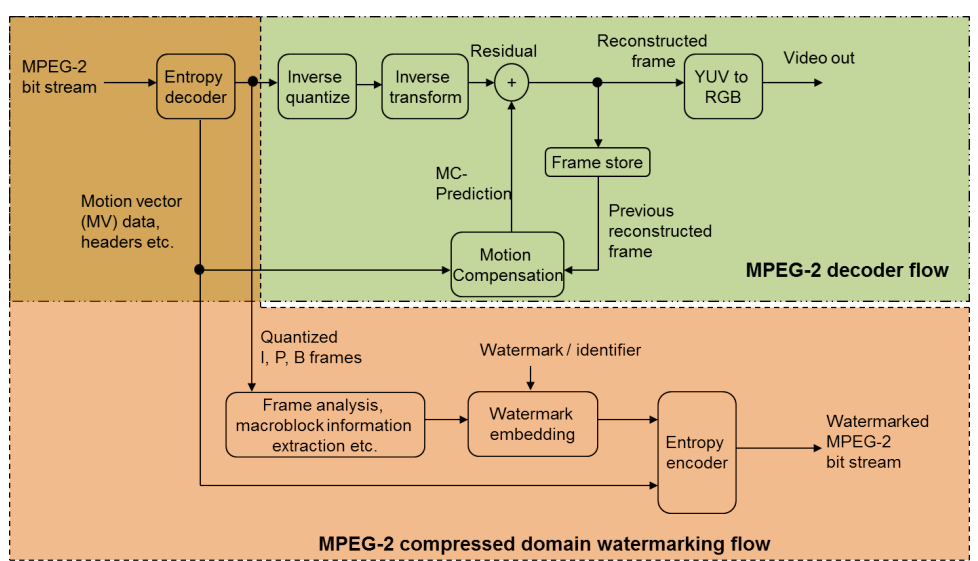

Fig. 3. Proposed MPEG-2 compression domain watermarking module.

\subsection{Watermarking}

Based on our statistical analysis of available video formats and industrial requirements (see Section 2.1), evidently, we concentrate on proposing watermarking algorithms for MPEG-2 and H.264 encoded videos. However to handle any other format we also propose a frame domain watermarking scheme that is robust to format changes and compression. In all cases we aim to preserve the quality of the assets closer to its original quality with minimum watermarking strengths. We also extract exact watermark sequence unlike traditional academically interesting correlation or similarity measure.

MPEG-2 compression domain watermarking We propose a new MPEG-2 compression domain watermarking module due to the widespread use of MPEG-2 video assets in industry. Our algorithm embeds watermark on a partially decoded MPEG-2 bit stream. Firstly the input bit stream is entropy decoded to produce quantized I, $P$ and $B$ frames. The motion vector (MV) data and other header information are kept separately. The partially decoded frames are then analysed and the quantized DC-DCT coefficients of the I frames are marked for watermark embedding. The least significant bits (LSB) of those DC coefficients are modified according to the incoming watermark bit. Once modified the frames are entropy encoded along with MV and header data to produce an MPEG-2 compatible watermarked bit stream. The overall functional block diagram of our proposed MPEG-2 compression domain watermarking module is shown in Fig. 3. Use of partial decoding within MPEG-2 flow allows us to avoid quality degradation due to re-compression. The extraction of watermark follows a similar partial decoding and collects the LSB of the DC coefficient as the extracted watermarking bit.

Zero-distortion H.264 compression domain watermarking Next we propose a zerodistortion H.264 compression domain watermarking scheme. This module embeds the watermark sequence within the Network Abstraction Layer (NAL) of the H.264 coded bit stream. The H.264 NAL standard defines 22 (/32) bits for various header information whereas $23^{\text {rd }}$ and $24^{\text {th }}$ bits are reserved and $25-32^{\text {nd }}$ bits are unspecified. We have used 3 bits of the unspecified NAL unit bits for embedding. The bits are altered according 
to the incoming watermarking bit sequence: 010 and 001 for 1 and 0 watermark bits, respectively. Further a sequence (110) is used for synchronisation after embedding of every watermark key. This allows to embed the watermark information within H.264 header without distorting the media content, and hence called zero-distortion watermarking. This approach provides a fast watermarking method which is robust to H.264 synchronisation attack and any H.264 file editing that preserves the header information.

Frame domain watermarking Finally we propose a frame domain watermarking scheme that is compatible to many video formats and robust to format changes \& reasonable compression. Although many joint watermarking-compression domain algorithms were proposed in the literature (e.g., for MPEG-2 [5] and H.264 [9]), any compression domain watermarking scheme is vulnerable to format changes which is one of the requirements in our case. Therefore, we propose a frame-domain watermarking algorithm where the media bit stream is first uncompressed to individual frames, preserving all encoding related information including information on interlacing for MPEG-2 videos. Further we propose a new discrete wavelet transform (DWT) domain watermarking scheme as the wavelet-based algorithms demonstrated superior performances in recent literature [3].

Considering the importance of imperceptibility, we propose a novel texture based watermarking algorithm as studies [2] suggest embedding within a textured region is far less noticeable compared to embedding in homogeneous regions. Our approach also considers identifying high frequency textured regions within the scene using DWT for watermark embedding due to DWT's dominance as a powerful tool for texture analysis [13]. DWT decomposes an image into independent frequency sub-bands of multiple orientations at multiple scales demonstrating details and structures.

Once wavelet decomposed the vertical and horizontal high frequency sub-bands are divided in multiple non-overlapping blocks of size $N \times N$. The cumulative energy of the blocks for a vertical sub-band are compared with cumulative energy of the corresponding blocks in the horizontal sub-band. Depending on the incoming watermark sequence $(W)$ the sub-band energies in pairs of blocks are modified so that the energy of one block is greater than the other and vice versa. This is achieved through modifying the coefficient values with a predefined strength parameter $(\alpha)$. This allows minimum distortion after embedding with reasonable robustness performance. Inverse DWT is then applied to reconstruct the watermarked frame. Finally these frames are encoded in the same format as received using the preserved parameter information to comply with the existing infrastructure. The algorithmic description of this module is shown in Algorithm 1. A blind extractor can extract the watermark information very quickly by comparing the block energies after wavelet decomposition of the test frame.

\subsection{Synchronisation}

Due to media post processing, e.g., scene editing, video summarising for trailer preparation, often multiple clips are created from a single media source or combined to create a single clip. It is important to identify the origin of composite media files consisting of multiple clips edited together. Therefore synchronisation at frame level or stream level for watermark identifier is necessary in this work. Our solution proposes two different synchronisation approaches to address this issue: 


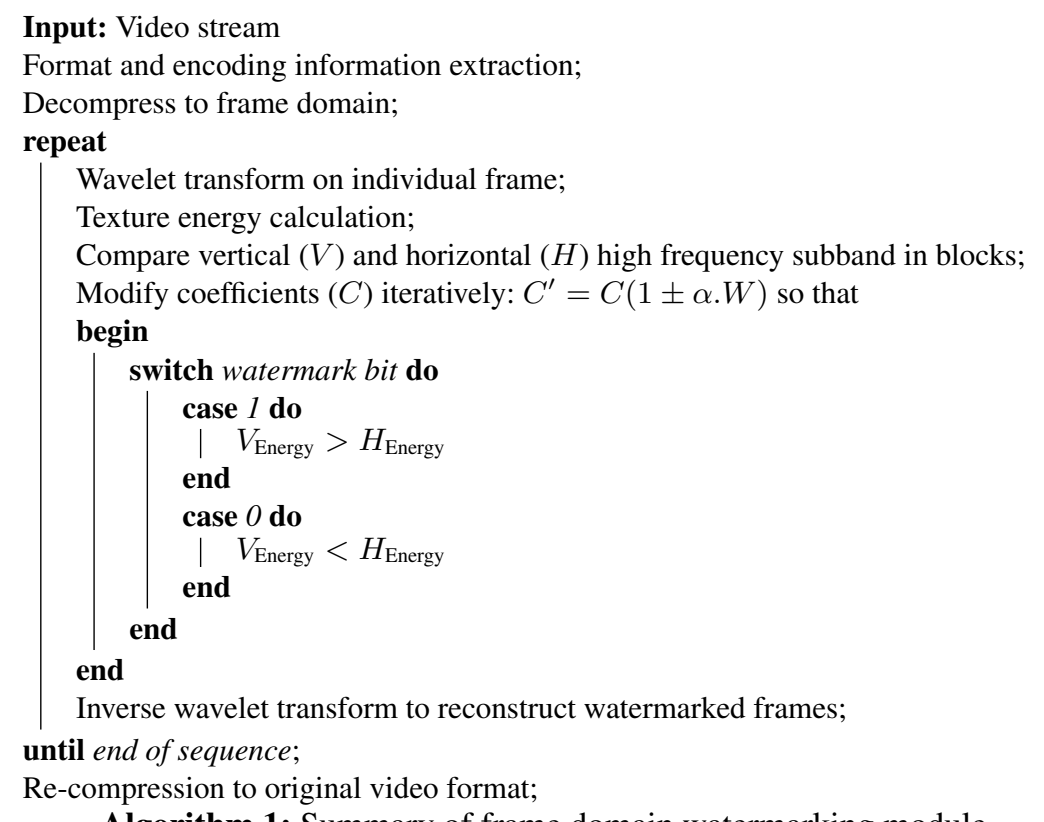

Algorithm 1: Summary of frame domain watermarking module.

Frame domain unique watermarking: During the frame domain watermarking, video streams are uncompressed to frame level and unique watermark identifiers are embedded in each frame. The modularity of the unique identifier relies on the target usage. For example, frames from multiple sources can be identified by embedding one identifier in every frame for each source. Different identifiers are extracted during authentication which also indicates the temporal location of various clips. In another scenario when frame dropping or frame editing needs to be tracked, each frame from single source requires embedding of a common identifier.

H.264 bit synchronisation: The proposed framework provides additional synchronisation for H.264 compressed domain watermarking (see Section 3.2). During the identifier embedding we add synchronisation bits every time. This self-synchronising method allows the user to detect any cropping in temporal domain or presence of multiple clips (from different sources) in H.264 domain.

\subsection{Overall watermarking work flow}

The overall flow diagram for embedding is shown in Fig. 2. During embedding, when a input media is received it is checked for format compatibility followed by a demultiplexing of video and audio files. The audio files are kept temporarily. In the case of MPEG-2 or H.264 video stream, one can choose either a frame domain watermarking, should the requirement be for robustness. Alternatively, for fast but less robust watermarking, the MPEG-2 or H.264 compression domain watermarking scheme can be chosen. For any other formats, frame domain watermarking is recommended. As a first step of the frame 
domain watermarking, the video stream is first uncompressed and compression parameters, available from header information, are kept. Once the watermark embedding is done the parameters are used to re-compress the media in the similar format and quality as it was received. Finally the re-multiplexing module combines video and audio to produce the final trackable media asset. Extraction flow is very similar to the embedding flow except, in this case we do not need to store any temporary audio file or other parameters. Once the watermark extraction is done, an XML report is generated to collect the overall statistics of the extracted watermark / identifier. This identifier is then sent to remote database for validation and metadata extraction as shown in Fig. 1.

\section{System Verification, Results and Discussions}

The interoperability of our solution was tested successfully and incorporated with an industrial media asset management system. Performance of individual modules is reported here using an exemplar test sequence $(\mathrm{Crew})^{5}$ with a dimension of $352 \times 288$. Firstly the uncompressed YUV sequence is encoded with 1) MPEG-2 compression and wrapped in a .MPG format and 2) H.264 compression and wrapped in a .MP4 format using FFmpeg to experiment with MPEG-2 compression domain and H.264 compression domain watermarking, respectively. Finally, both the formats are used to perform frame domain watermarking and tested against the requirements set out in the beginning of this paper. The proposed system is also verified with more sequences (available from the industrial partners' repository) with various media formats including, MPEG2, H.264, ProRes, DPX and MJPEG.

In this experimental set up for MPEG-2 watermarking, we have chosen I-frame only embedding as that is more likely robust compared to $\mathrm{P}$ or $\mathrm{B}$ frame embedding. The H.264 watermarking does not need user defined parameter as the module is restricted to header modification within the NAL unit to provide a zero-distortion embedding mechanism. Finally, the frame domain watermarking considers a computationally inexpensive lifting based bi-orthogonal $5 / 3$ wavelet kernel with one level decomposition and a block size of $4 \times 4$ (i.e., $N=4$ ). The watermark consists of a sequence that represents a 64-bit binary identifier.

\subsection{Results and Discussion}

In verifying the compatibility with various media formats, the proposed solution is tested with various media wrappers and successfully de-multiplexed video and audio streams separately. Once the video streams are watermarked using one of the three available modules, it multiplexed the watermarked video stream with temporarily stored audio file to output in a format same as the input one. We also compare the compatibility with video stream formats, e.g., MPEG-2 watermarking is only usable for MPEG-1 or MPEG-2 compatible streams, H.264 watermarking is usable with .264 complied bit stream while frame domain watermarking can be used for a wide range of video stream formats. Currently supported video stream formats for frame domain watermarking include MPEG-2, H.264, ProRes, DPX and MJPEG. Interlaced videos commonly available with many existing MPEG-2 streams for broadcasting are also supported in the proposed solution. A summary of media format compatibility is shown in Table 2.

\footnotetext{
${ }^{5}$ https://media.xiph.org/video/derf/
} 


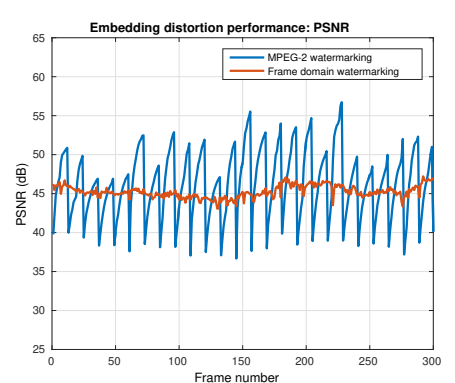

(a) PSNR plot.

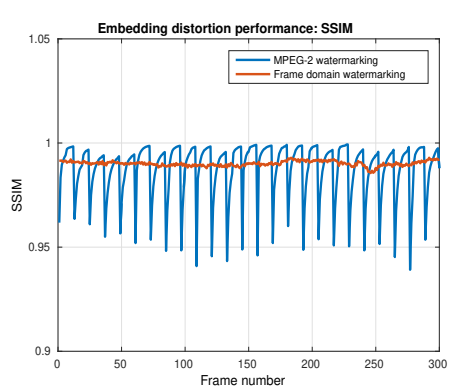

(b) SSIM plot.

Fig. 4. Embedding distortion performance of MPEG-2 compression domain and frame domain watermarking for Crew test sequence: (a) Average PSNR: 46.24dB (MPEG-2) \& 45.23dB (frame domain) and (b) Average SSIM: 0.99 (MPEG-2) \& 0.99 (frame domain).

Media quality has major influence in the design of our solution. In this work we used two existing objective measurements: Peak Signal to Noise Ratio (PSNR) and Structural Similarity Index Measure (SSIM) [14], to quantify any distortion due to watermark embedding. In both cases a higher value indicates superior performances. Generally PSNR value more than $35 \mathrm{~dB}$ and SSIM more than 0.9 indicate imperceptible embedding performances. The embedding distortion performances, i.e., PSNR and SSIM for MPEG-2 and frame domain watermarking are shown in Fig. 4(a) and Fig. 4(b), respectively. The $\mathrm{x}$-axis in each plot indicates the distortion measure and $\mathrm{y}$-axis represents the frame number. In both cases the results show PSNR values well above $35 \mathrm{~dB}$ with an average of $46.24 \mathrm{~dB}$ (MPEG-2) and $45.23 \mathrm{~dB}$ (frame domain); and SSIM values above 0.9 with an average of 0.99 in both cases. The ripple effect in MPEG-2 watermarking is due to I-frame only embedding and subsequent error propagation. However due to high PSNR / SSIM value distortions are not noticeable. It is worth noting that we do not report the embedding performance for H.264 watermarking as that does not modify the media content, hence called zero-distortion watermarking.

The robustness performances against MPEG-2 and H.264 compression are reported in Table 2. New compressed test sequences are obtained by setting the quantisation parameters in both compression standards, i.e., the quality scales (Q Scale) for MPEG-2 and the quantisation parameter (QP) for H.264. Maximum value of Q Scale $=4$ and QP $=20$ were set based on the current practices in DVD quality content production (hence we refrained from reporting robustness results at higher compression ratio). Extraction is applied on these compressed test sequences to retrieve the exact watermark. Our results show superior performance by the frame domain watermarking while MPEG-2 watermarking performs within reasonable expectations. H.264 watermarking in compression tests failed in all cases as compression involves decoding and re-encoding of the media where the header information is lost. However the latter one is the fastest to compute among three (frame domain being slowest) and is useful to handle synchronisation and media clip identification. We avoid reporting the robustness results for signal processing watermarking attacks, such as filtering, noise inclusion etc. as these are not part of the requirements and hence considered outside the scope of this paper. 


\begin{tabular}{|c|c|c|c|}
\hline \multirow{2}{*}{ Compatibility $\Downarrow$} & \multicolumn{3}{|c|}{ Watermarking Schemes } \\
\hline & $\left|\begin{array}{c}\text { MPEG-2 } \\
\text { based }\end{array}\right|$ & $\left|\begin{array}{l}\mathbf{H} .264 \\
\text { based }\end{array}\right|$ & $\begin{array}{c}\text { Frame domain } \\
\text { (preferred solution) }\end{array}$ \\
\hline \multicolumn{4}{|c|}{ Media format compatibility } \\
\hline AV media wrapper (DAT, MOV, AVI, VOB, MP4) & $\checkmark$ & $\checkmark$ & $\checkmark$ \\
\hline Video stream: MPEG-2 (including interlacing) & $\checkmark$ & $x$ & $\checkmark$ \\
\hline Video stream: H.264 & $x$ & $\checkmark$ & $\checkmark$ \\
\hline Video stream: ProRes, DPX, M-JPEG etc. & $x$ & $x$ & $\checkmark$ \\
\hline \multicolumn{4}{|c|}{ Robustness against compression } \\
\hline MPEG-2 compression (Q Scale = 2) & $\checkmark$ & $x$ & $\checkmark$ \\
\hline MPEG-2 compression (Q Scale = 4) & $x$ & $x$ & $\checkmark$ \\
\hline H.264 compression $(\mathrm{QP}=20)$ & $x$ & $x$ & $\checkmark$ \\
\hline \multicolumn{4}{|c|}{ Synchronisation } \\
\hline Joining \& splitting multiple clips identification & $x$ & $\checkmark$ & $\checkmark$ \\
\hline Frame inclusion \& dropping detection & $x$ & $x$ & $\checkmark$ \\
\hline
\end{tabular}

Table 2. Compatibility of PARTEC to industrial requirements.

We report the results satisfying the requirements of synchronisation as described in Section 2.1. The proposed scheme can detect sources of multiple video clips (scene) joined together during scene editing or tracking multiple clips generated from a single source. The solution is also capable to identify frame dropping, frame inclusion within a sequence using frame level watermark synchronisation (frame domain watermarking) or header based synchronisation (H.264 watermarking). A summary of the ability to synchronise for three different watermarking modules are shown in Table 2.

Finally we report the level of complexity for our watermarking module. H.264 only modify header information within H.264 bit stream and hence requires least amount of computation, whereas MPEG-2 watermarking module partially decode, watermark and re-encode the media and has reasonable complexity. The frame domain watermarking satisfies all requirements of PARTEC and robust against compression but exhibits higher computational complexity during watermark embedding. However, the extraction procedure is highly efficient providing opportunity for real-time performance.

\section{Conclusions}

PARTEC proposed a new application of watermarking techniques for the purpose of assets tracking and authentication. It considered wider industry requirements in both user (media or content post processing companies) and developers (of media asset management systems) point of view. PARTEC developed the watermarking based solution by analysing industrial requirements and produced a system that is compatible with the existing infrastructure. The requirements were dissected in four different categories, e.g., 
media format compatibility, imperceptibility, robustness and synchronisation. Three different watermarking algorithms were proposed in this work: a) MPEG-2 compression domain, b) zero-distortion H.264 compression domain and c) wavelet-based frame domain watermarking. Experimental verification showed promising results for the target application. We conclude that our approach with the frame domain video watermarking is format agnostic and suitable to fulfil the requirements listed in this work.

Acknowledgment: We acknowledge the support of Innovate UK (Project Ref: 100946).

\section{References}

1. Asikuzzaman, M., Alam, M.J., Lambert, A.J., Pickering, M.R.: Imperceptible and robust blind video watermarking using chrominance embedding: A set of approaches in the dt cwt domain. IEEE Trans. on Information Forensics and Security 9(9), 1502-1517 (2014)

2. Barni, M., Bartolini, F., Piva, A.: Improved wavelet-based watermarking through pixel-wise masking. IEEE Trans. Image Processing 10(5), 783-791 (May 2001)

3. Bhowmik, D., Abhayaratne, C.: Quality scalability aware watermarking for visual content. IEEE Transactions on Image Processing 25(11), 5158-5172 (2016)

4. Bianchi, T., Piva, A.: Secure watermarking for multimedia content protection: A review of its benefits and open issues. IEEE Signal Processing Mag. 30(2), 87-96 (2013)

5. Biswas, S., Das, S.R., Petriu, E.M.: An adaptive compressed mpeg-2 video watermarking scheme. IEEE Trans. on Instrumentation and Measurement 54(5), 1853-1861 (Oct 2005)

6. Chan, H.T., Hwang, W.J., Cheng, C.J.: Digital hologram authentication using a hadamardbased reversible fragile watermarking algorithm. Journal of Display Technology 11(2), 193203 (2015)

7. Cox, I.J., Miller, M.L., Bloom, J.A.: Watermarking applications and their properties. In: Information Technology: Coding and Computing, 2000. Proceedings. International Conference on. pp. 6-10 (2000)

8. Fallahpour, M., Shirmohammadi, S., Semsarzadeh, M., Zhao, J.: Tampering detection in compressed digital video using watermarking. IEEE Trans. on Instrumentation and Measurement 63(5), 1057-1072 (2014)

9. Mansouri, A., Aznaveh, A.M., Torkamani-Azar, F., Kurugollu, F.: A low complexity video watermarking in h.264 compressed domain. IEEE Trans. on Information Forensics and Security 5(4), 649-657 (2010)

10. Nezhadarya, E., Wang, Z.J., Ward, R.K.: Image quality monitoring using spread spectrum watermarking. In: Proc. IEEE ICIP. pp. 2233-2236 (2009)

11. Stütz, T., Autrusseau, F., Uhl, A.: Non-blind structure-preserving substitution watermarking of H.264/CAVLC inter-frames. IEEE Trans. on Multimedia 16(5), 1337-1349 (2014)

12. Tsai, J.M., Chen, I.T., Huang, Y.F., Lin, C.C.: Watermarking technique for improved management of digital medical images. Journal of Discrete Mathematical Sciences and Cryptography 18(6), 785-799 (2015)

13. Ves, E., Acevedo, D., Ruedin, A., Benavent, X.: A statistical model for magnitudes and angles of wavelet frame coefficients and its application to texture retrieval. Pattern Recognition 47(9), 2925 - 2939 (2014)

14. Wang, Z., Bovik, A.C., Sheikh, H.R., Simoncelli, E.P.: Image quality assessment: From error visibility to structural similarity. IEEE Trans. Image Processing 13, 600-612 (2004)

15. Yamada, T., Maeta, M., Mizushima, F.: Video watermark application for embedding recipient ID in real-time-encoding VoD server. J. of Real-Time Image Proc. 11(1), 211-222 (2016)

16. Zhang, H., Shu, H., Coatrieux, G., Zhu, J., Wu, Q.M.J., Zhang, Y., Zhu, H., Luo, L.: Affine legendre moment invariants for image watermarking robust to geometric distortions. IEEE Trans. on Image Processing 20(8), 2189-2199 (2011) 